\title{
Psicosis cicloides: Controversias diagnósticas y nosológicas a propósito de un caso
}

\begin{abstract}
RESUMEN: Revisión histórica sobre la psicosis cicloide y su ubicación nosológica, así como sus relaciones con las dos grandes psicosis endógenas kraepelinianas y otras categorías como las polisémicas psicosis agudas.

PALABRAS CLAVE: Psicosis cicloide, psicosis aguda, esquizofrenia, trastorno bipolar.
\end{abstract}

\begin{abstract}
SUMMARY: Historic review of the controversial concept of cycloid psychosis and its nosologic location, as well as its relationships with the two other kraepelinian endogenous psychosis and the polysemous acute psychosis.

KEY WORDS: Cycloid psychosis, acute psychosis, schizophrenia and bipolar disorder.
\end{abstract}

El desempeño diario de la actividad clínica en el campo de la salud mental está marcado, en la actualidad, por el uso de dos manuales clasificatorios sobradamente conocidos: la CIE-10 (1) y el DSM-IV-TR (2). No es el propósito del presente trabajo negar su indudable utilidad al conseguir una cierta equiparabilidad entre los diagnósticos realizados por profesionales en distintas partes del mundo, pero también es cierto que han sido objeto de distintas críticas desde posiciones diversas. En el presente trabajo nos proponemos, partiendo del comentario de un caso clínico visto por nosotros, revisar histórica y conceptualmente el diagnóstico de psicosis cicloide que, como tal, no viene recogido en ninguno de estos sistemas. Según las clasificaciones de la OMS y la APA, no existe este diagnóstico y es necesario ubicar a los pacientes en otras categorías, como luego veremos, perdiéndose en nuestra opinión ciertas características y diferencias importantes entre cuadros parecidos. Traemos este paciente en concreto queriendo destacar sobre todo que en absoluto constituye un caso extraordinario, sino más bien al contrario, un ejemplo de muchos casos similares que creemos podrían encuadrarse dentro de esta categoría de las psicosis cicloides, lo que evitaría tener que forzar su inclusión en otras, con implicaciones a nivel de pronóstico o tratamiento que podrían ser importantes.

\section{Caso clínico}

Nuestro paciente es un varón que tiene en la actualidad 32 años. Es soltero, sin hijos, el primero de tres hermanos. Cuenta con estudios superiores de Física y trabaja actualmente como profesor de Secundaria, habiéndose independizado recientemente de casa de sus padres al empezar a trabajar. Sus antecedentes psiquiátricos comienzan en julio de 2001, a los 28 años de edad, momento en que es 
valorado por un psiquiatra por «estrés laboral», sin que se pusieran de manifiesto en la exploración psicopatológica síntomas psicóticos ni de otra naturaleza, no indicándose tratamiento ni seguimiento alguno. Se produce una nueva consulta en enero de 2002 donde se diagnostica un trastorno psicótico de pocos meses de evolución, sin que quede claro exactamente de cuánto tiempo estamos hablando, aunque reconstruyendo posteriormente la historia con la familia pudo tratarse sólo de unas semanas. Se describe un delirio de perjuicio, con múltiples interpretaciones delirantes del entorno y vivencias autorreferenciales. El paciente se encuentra hipotímico aunque no llega a presentar un síndrome depresivo completo y tiene una parcial conciencia de enfermedad. No existen antecedentes personales relevantes ni desencadenantes ambientales aparentes. En ese momento, vive con padres y hermanos, sin conflictos reseñables, con adecuada adaptación al medio social y contando con relaciones personales aparentemente normales. No consta que haya habido consumo de tóxicos ni tampoco aparecen antecedentes familiares de enfermedades mentales. El diagnóstico en ese momento es de trastorno psicótico agudo y se trata con 4,5 mg/día de risperidona. Al mes de diagnosticarse el cuadro la mejoría es muy evidente, persistiendo sólo ciertas vivencias autorreferenciales ante las que el paciente admite la crítica. Tras tres meses de seguimiento ambulatorio se llega a una remisión completa de la sintomatología psicótica.

En mayo de 2004 tiene un nuevo episodio, siendo controlado en esa ocasión por un psiquiatra privado, también de forma ambulatoria como la primera vez. La sintomatología, según la información facilitada por el mismo paciente y su familia a posteriori, era muy similar al primer cuadro descrito. Es tratado con perfenazina (6 mg/día) y amitriptilina (30 mg/día) consiguiéndose en pocas semanas una remisión completa ad integrum. Mantiene dicho tratamiento durante cinco meses y luego lo interrumpe, permaneciendo completamente asintomático hasta marzo de 2005, momento en que tiene su primer ingreso. Comienza a presentar de forma brusca, una semana antes de dicho ingreso, inquietud, ansiedad intensa, insomnio y soliloquios, con suspicacia y negativa a la ingesta de alimentos, llegando a mostrarse agresivo verbalmente hacia su familia. Se pone de manifiesto ideación delirante de perjuicio centrada en su entorno sociofamiliar, con vivencias autorreferenciales marcadas, así como un afecto un tanto expansivo. Aunque los primeros días se encuentra colaborador, de forma súbita se muestra irritable, con agresividad contenida, aunque también en pocos días esto cede y pasa a estar correcto y adecuado, eutímico, llegando a hacer crítica de sus síntomas psicóticos y alteraciones de conducta. La familia opina que está totalmente recuperado, siendo ésa también nuestra impresión, por lo que procedemos al alta. El ingreso dura tres semanas, tras las cuales el paciente toma $7,5 \mathrm{mg} /$ día de risperidona, que progresi- 
vamente va disminuyéndose de forma ambulatoria, llegando a suspenderse completamente dado que el paciente continúa asintomático, habiéndose reintegrado a su actividad laboral sin ningún problema. El diagnóstico es de trastorno psicótico agudo polimorfo sin síntomas de esquizofrenia.

En febrero de 2006 ocurre un nuevo episodio, similar a los previos, que requiere ingreso. El paciente está bien hasta dos días antes de acudir al hospital, iniciándose de forma brusca un cuadro consistente en insomnio total, inquietud psicomotriz y heteroagresividad verbal en su entorno, presentando ideación delirante de perjuicio y megalomaniaca, vivencias autorreferenciales y alucinaciones auditivas en forma de voces imperativas. El ingreso dura dos semanas, observándose la misma variación en la actitud del paciente que en el previo: muy colaborador los primeros días, francamente hostil y amenazante luego para, de forma brusca, mostrar una conducta adecuada y una plena crítica de sus ideas delirantes, aunque mostrándose un tanto reacio a hablar de ellas. El afecto, en esta ocasión, no ha sido particularmente expansivo como en el ingreso previo. El tratamiento al alta es de amisulpride ( $800 \mathrm{mg} /$ día), siendo diagnosticado también de trastorno psicótico agudo polimorfo sin síntomas de esquizofrenia. El seguimiento ambulatorio confirma que la remisión ha sido completa y el paciente ha vuelto a su actividad laboral, sin que se observe ningún deterioro en sus relaciones interpersonales ni en ningún otro aspecto.

\section{Revisión biliográfica}

Llevaremos ahora a cabo una revisión de la bibliografía que, como todas, será siempre parcial, pero que creemos necesaria para intentar llegar a un diagnóstico certero del paciente.

En primer lugar, los cuatro episodios que ha presentado han sido diagnosticados de trastorno psicótico agudo y transitorio. Es esta una categoría recogida en la CIE-10 que supone un comienzo agudo en menos de dos semanas desde un estado previo no psicótico a otro con sintomatología delirante o alucinatoria, así como alteraciones conductuales y otros síntomas posibles como, por ejemplo, lenguaje disgregado. Puede haber cambios emocionales o síntomas afectivos ocasionalmente en primer plano, pero sin llegar a configurar un episodio maníaco o depresivo. Cuando la sintomatología es rápidamente cambiante y variable se usa el subtipo de «polimorfo». Es posible que haya habido presencia de estrés agudo previo o no y la evolución será hacia una recuperación completa en un plazo no determinado de forma concreta (menos de dos o tres meses aproximadamente). Los cuadros con estas características son diagnosticados según los criterios del DSM-IV-TR 
como trastorno psicótico breve si la duración es menor de un mes y como trastorno esquizofreniforme si es mayor de un mes pero menor de seis.

El diagnóstico transversal de cada uno de los episodios del paciente correspondería a lo que clásicamente se han llamado psicosis agudas, tema que revisa de forma magistral Luque (3) en un trabajo reciente. Como este autor señala, son cuadros psicóticos de comienzo súbito, duración breve y remisión completa. Clínicamente pueden aparecer delirios polimorfos, alucinaciones de cualquier tipo, variaciones emocionales y del comportamiento, síntomas psicomotores y conductas extrañas, así como un lenguaje fragmentado y desordenado. El pronóstico es bueno ya que el episodio remite por completo en casi todos los casos, recuperándose el nivel de funcionamiento premórbido y permaneciendo la personalidad intacta. Estos trastornos han recibido múltiples denominaciones por parte de distintos autores que se han aproximado a estas psicosis agudas desde diversos puntos de vista.

Valentin Magnan (4), como recoge Luque, crea el concepto de bouffée délirante, una de las referencias fundamentales de la psiquiatría francesa. Estos cuadros estaban ligados a la idea de «degeneración» de Morel, que hace referencia a una determinada predisposición o diátesis que conlleva la posible aparición de estas psicosis. El concepto de degeneración no se refiere a que vaya a producirse un deterioro en el paciente, sino sólo a esta predisposición que implicará riesgo de recidivas. Se trataba de episodios psicóticos de comienzo súbito, constituyendo cuadros delirantes polimorfos, variables y pluritemáticos, con posible enturbiamiento de la conciencia e inestabilidad emocional, de evolución rápida y finalización brusca con recuperación completa. Aunque las recidivas son posibles, los intervalos no presentan síntomas. El diagnóstico de bouffée délirante cayó en desuso por el predominio de la nosología de Kraepelin (5) y por el amplio concepto de esquizofrenia de Bleuler (6) que igualaba estos cuadros con las llamadas esquizofrenias agudas.

La recuperación de la bouffée délirante la llevó a cabo Henri Ey $(7 ; 8)$, quien las describe, siguiendo a Magnan, como cuadros caracterizados por la eclosión súbita de un delirio transitorio y polimorfo en sus temas y manifestaciones, rico en intuiciones e interpretaciones delirantes, alucinaciones diversas y alteraciones del humor, que puede oscilar de la exaltación a la angustia. Henri Ey ordena las enfermedades mentales en agudas, clasificadas como alteraciones de la conciencia, y, crónicas, clasificadas como alteraciones de la personalidad. Las bouffées délirantes serían formas delirantes agudas opuestas a los delirios crónicos. La descripción que hace Ey de estas psicosis agudas es difícilmente superable: «[...] El delirio es polimorfo, es decir que sus temas son múltiples y variables: de persecución, de grandeza, de transformación sexual, de posesión, de envenenamiento, de 
influencia, de riqueza o de fabulosa potencia, etc. Generalmente se imbrican, se mezclan y sufren metamorfosis, como en la sucesión caleidoscópica de las imágenes oníricas [...]. El carácter polimorfo de este delirio episódico se manifiesta también en la yuxtaposición de los fenómenos que lo componen. Clásicamente, se distinguen sobre todo convicciones e intuiciones que irrumpen en el psiquismo [...]. Pero las alucinaciones son numerosas y exuberantes, con frecuencia auditivas, pero más generalmente psíquicas (voz, eco del pensamiento, inspiraciones, actos impuestos, etc.). Están asociadas a interpretaciones delirantes, a elementos imaginativos, ilusiones, sentimientos e impresiones que manifiestan la incoercibilidad de la experiencia delirante vivenciada, en una atmósfera de misterio y apocalipsis. Las variaciones del cuadro clínico son características de esta rica diversidad de episodios que se imbrican y se suceden. El enfermo presenta importantes cambios de humor y violentas oscilaciones [...]. El humor está alterado de manera constante. A la actividad delirante de aparición súbita corresponden, en efecto, violentos estados afectivos. Unas veces el sujeto está exaltado y expansivo como un maníaco. Otras, por el contrario, se halla preso de gran angustia, más o menos próxima a la experiencia melancólica [...]. Esta alternancia o esta combinación de excitación e inhibición es tan característica de estos brotes delirantes polimorfos que $[\ldots]$ hace que en la clínica sea a veces difícil establecer un diagnóstico diferencial entre una bouffée délirante y una crisis maniacodepresiva». En cuanto al pronóstico, Ey afirma que se llega a una remisión sin secuelas ni complicaciones, aunque, al suponer que el paciente tiene una predisposición a estos cuadros, existe riesgo de recidiva. También es posible que algunos casos de psicosis delirantes agudas terminaran evolucionando a una psicosis esquizofrénica o a un delirio crónico del tipo de la paranoia o la parafrenia. En el sistema clasificatorio actual empleado en la psiquiatría francesa, el INSERM (9), todavía se emplea el concepto de bouffée délirante, en base a unos criterios operacionales que siguen las descripciones clásicas.

Otra denominación que han recibido históricamente las psicosis agudas ha sido el de psicosis psicogénicas, empleado hoy en día como prácticamente sinónimo de psicosis reactivas. En un intento de definición sencillo pero eficaz, Sommer (10), como cita Luque, distingue a las enfermedades psicogénicas como «estados mentales patológicos que pueden evocarse y ser influidos por las ideas». Estas psicosis fueron estudiadas en profundidad por Wimmer (11) que definió a las psicosis psicogénicas como un grupo de psicosis clínicamente independientes causadas por «traumas psíquicos» en sujetos con una «predisposición psicopática». Strömgren $(12 ; 13)$ ha defendido la independencia nosológica de estas psicosis, aduciendo una serie de razones: el pronóstico de las psicosis reactivas es bueno comparado con la esquizofrenia o el trastorno bipolar y, aunque puede 
haber recidivas, no son tan frecuentes como en este último. Además, Strömgren comenta que el tratamiento de elección en estas psicosis sería la psicoterapia de apoyo, que podría combinarse brevemente con ansiolíticos, llegando a afirmar que un tratamiento prolongado con neurolépticos, como si se tratase de una esquizofrenia, sería innecesario y provocaría efectos secundarios sin beneficio alguno. También este último autor ha establecido una serie de características de las llamadas psicosis psicogénicas: el trauma es determinante para que aparezca la psicosis; existe una relación temporal entre ambos; el trauma influye en el contenido de los síntomas psicóticos; hay una vulnerabilidad en la personalidad del sujeto que predispone a la psicosis; si el trauma desaparece la psicosis también lo hace, pero aunque aquél persista, la psicosis termina remitiendo; lo que determina la psicosis es la experiencia subjetiva al significado del trauma, no la intensidad objetiva del mismo; por último, la imposibilidad de establecer la existencia de un trauma no es causa suficiente para descartar el diagnóstico de psicosis psicogénica.

Otra categoría que encuadra trastornos dentro del ámbito de las psicosis agudas son las denominadas y siempre polémicas psicosis histéricas, estrechamente relacionadas con las psicosis psicogénicas o reactivas y frecuentemente confundidas con ellas. Como señala Luque, Kraepelin admitía la aparición de síntomas similares a los psicóticos en la histeria, mientras que Bleuler afirmaba tajantemente: «Nunca tuve motivo alguno para hacer un diagnóstico de psicosis histérica. Todos los casos así diagnosticados no se distinguen de la esquizofrenia. Cuando un supuesto histérico se convierte en esquizofrénico o se deteriora, en mi experiencia no era un histérico sino un esquizofrénico». No debe olvidarse que para Bleuler el concepto de esquizofrenia incluía las llamadas esquizofrenias agudas, que serían asimilables a las psicosis agudas de otros autores. Faergeman (14) consideraba las psicosis histéricas como un subgrupo de las psicosis psicogénicas en las que existe una motivación para la sintomatología. Para Hollender y Hirsch (15), algunas de las características de las psicosis histéricas serían: comienzo súbito en relación con algún acontecimiento perturbador; alucinaciones, delirios, despersonalización y alteraciones conductuales; si la afectividad está alterada es inestable y voluble, no aplanada; duración entre una y tres semanas, con recuperación completa; personalidad previa con rasgos histéricos presentes. Modestin y otros (16), tras un estudio de seguimiento de varios años a un grupo de pacientes, concluyeron que la psicosis histérica no constituye una entidad clínica diferenciada del conjunto de las psicosis psicogénicas / reactivas.

Otro grupo de psicosis agudas descritas, u otra forma de denominar a cuadros tal vez muy similares, fueron las psicosis esquizofreniformes de Langfeldt (17). Son casos de comienzo agudo en relación con algún factor precipitante, en sujetos con personalidad previa ajustada, con síntomas similares a la esquizofrenia 
pero con frecuente obnubilación de la conciencia y depresión. Langfeldt relacionó estos cuadros con la constitución corporal pícnica. Como comenta Luque en su revisión, muchos autores han cuestionado la validez y la utilidad clínica de este diagnóstico.

Un término que ha llegado hasta nuestros días, aunque con un significado ostensiblemente modificado, es el de psicosis esquizoafectivas. Acuñado originalmente por Kasanin (18), hacía referencia a un cuadro psicótico observado en pacientes jóvenes, con gran labilidad emocional, síntomas esquizofrénicos y afectivos, grave distorsión de la percepción del mundo exterior y presencia de «impresiones sensoriales falsas». Estos enfermos se recuperaban completamente en pocas semanas, aunque había una clara tendencia a la recidiva.

Un autor que también ha dejado su aproximación a estos cuadros ha sido Castilla del Pino (19). Emplea la denominación de psicosis paranoide aguda y la define como «un cuadro clínico delirante alucinatorio, de carácter benigno, con tendencia a la curación sin secuelas, de evolución breve y que comienza y concluye de forma abrupta». Suele haber algún desencadenante y ciertos pródromos consistentes en desasosiego y angustia junto a intenso insomnio. Aparecen delirios polimorfos y pluritemáticos, alucinaciones auditivas, cenestésicas y visuales, alteraciones del discurso con disgregaciones e incoherencias, con un estado de ánimo marcado por la angustia o el éxtasis, con agitación o inhibición psicomotriz.

Hasta aquí, hemos realizado un breve recorrido por algunos de los cuadros descritos por distintos autores dentro del ámbito de las psicosis agudas, considerando que los cuatro episodios padecidos por nuestro paciente se podrían catalogar como tales. Otra cuestión diferente es si se puede hacer algún diagnóstico longitudinal en este caso o en otros similares. La gran división nosológica en el campo de las psicosis endógenas crónicas fue efectuada por Kraepelin, entre demencia precoz y psicosis maniaco-depresiva. El término de demencia precoz fue sustituido posteriormente por el de esquizofrenia, creado por Bleuler. Mientras que Kraepelin puso el acento en el curso longitudinal progresivo y deteriorante de la enfermedad para efectuar el diagnóstico, Bleuler incidió más en el estado transversal del paciente en un momento dado, poniendo de relieve la escisión entre pensamiento, emoción y comportamiento. Schneider (20), por su parte, describió los llamados síntomas de primer rango, muy útiles para el diagnóstico, pero no imprescindibles para el mismo ni tampoco totalmente exclusivos de la esquizofrenia. Los criterios actuales de la CIE-10 y el DSM-IV-TR recogen los síntomas positivos y negativos, así como cierto grado de disfunción social o laboral desde el comienzo del trastorno. La duración de estas alteraciones debe ser al menos de seis meses.

La otra gran psicosis endógena diferenciada por Kraepelin fue la psicosis maniaco-depresiva, hoy denominada trastorno bipolar, de curso periódico con 
fases de enfermedad caracterizadas por episodios depresivos, maniacos o mixtos. La CIE-10 señala que lo característico es que la recuperación sea completa entre los episodios, pero el DSM-IV-TR afirma que algunos pacientes muestran labilidad afectiva y otros síntomas residuales entre los episodios, pudiendo aparecer también problemas crónicos interpersonales o laborales. Tenemos también un cuadro especialmente controvertido desde el punto de vista nosológico, como es el trastorno esquizoafectivo, que emplea el mismo término creado por Kasanin para describir pacientes muy diferentes. El DSM-IV-TR lo describe como un trastorno con sintomatología de episodio depresivo, maniaco o mixto junto a síntomas característicos de esquizofrenia, sin que sea precisa la aparición de disfunción social o laboral, pero sí un periodo de al menos dos semanas con síntomas delirantes o alucinatorios pero no afectivos marcados. La CIE-10, por su parte afirma que el diagnóstico de trastorno esquizoafectivo debe hacerse cuando las manifestaciones de ambos tipos de síntomas, esquizofrénicos y afectivos, son claras y destacadas y aparecen simultáneamente durante el mismo episodio. No debe diagnosticarse en casos de esquizofrenia con episodios depresivos ocasionales, como por ejemplo en la depresión postesquizofrénica. La aparición de un episodio esquizoafectivo ocasional dentro de un trastorno bipolar no invalida este diagnóstico. También afirma la CIE-10 que los pacientes con episodios esquizoafectivos recurrentes generalmente se recuperan de forma completa y sólo rara vez desarrollan un estado defectual. Por otra parte, la paranoia o trastorno de ideas delirantes se caracteriza fundamentalmente por ideas delirantes con muy escaso componente alucinatorio y sin deterioro salvo el directamente provocado en el desempeño interpersonal y social del paciente como consecuencia de su actividad delirante. Además, esta ideación debe durar como mínimo tres meses según la CIE-10 o un mes según el DSM-IV-TR.

En nuestra opinión, el paciente no cumple criterios para el diagnóstico de ninguno de estos trastornos. En ocasiones ha presentado un afecto exaltado y maniforme, pero de escasa duración y no parece que los síntomas afectivos hayan sido predominantes en ninguno de los episodios, apareciendo en primer lugar y de forma más llamativa la sintomatología delirante y alucinatoria, aunque siempre con recuperación completa en poco tiempo y sin secuelas posteriores de ningún tipo. Como hemos visto también repetidas veces en la revisión de las psicosis agudas, dentro de estos cuadros es frecuente la aparición de ánimo exaltado que puede fluctuar con la angustia.

Existe otro diagnóstico, descrito por autores clásicos y contemporáneos pero fuera como tal de las clasificaciones actuales, que es el de psicosis cicloide, con las características de algunas de las psicosis agudas que ya hemos visto más la insistencia en el aspecto del curso longitudinal, marcado por las recurrencias sin defecto. En nuestro entorno, ha sido Barcia $(21 ; 22)$ quien más ha insistido en rei- 
vindicar la importancia de este tipo de trastorno psicótico. Siguiendo a este autor, puede describirse el desarrollo histórico de estas psicosis desde Kleist (23), quien desarrolló el concepto de psicosis marginales, a partir de los cuadros descritos por distintos autores, como las psicosis de la motilidad o la psicosis de angustia de Wernicke, la paranoia primaria aguda de Westphal o la confusión alucinatoria de Krafft-Ebing, así como los conceptos de bouffée délirante de Magnan y Legrain y de psicosis periódica de la psiquiatría alemana de Schröder y Binswanger, entre otros autores. Kleist describió este grupo de psicosis, postulando la idea de una predisposición en forma de «constitución autóctona lábil», como reelaboración de la tesis de la degeneración. El término psicosis marginales hace referencia a que estaban en relación con las otras psicosis endógenas de la nosología kraepeliniana, pero sólo respecto a algunos rasgos y síntomas, ya que Kleist consideraba que constituían un grupo autónomo, que dividió en cinco subtipos.

Como señala Barcia, los planteamientos de Kleist fueron seguidos por Leonhard $(24 ; 25)$, quien propuso el nombre de psicosis cicloides para todo el grupo, empleando el término que Kleist había usado para uno de sus subtipos. Leonhard habló de tres tipos: la psicosis de angustia-felicidad, la psicosis confusional (incoherente-estuporosa) y la psicosis de la motilidad (acinética-hipercinética).

La clínica de estos cuadros tiene, como recoge Barcia, algunos rasgos fundamentales. Es característico el polimorfismo, que hace referencia a la gran variabilidad sintomatológica, tanto dentro de un mismo episodio como entre unos episodios y otros. Hay una alteración global de la vida psíquica, lo que hizo que fueran denominadas holodisfrenias por Barahona Fernandes, modificándose en pocos días de manera radical el pensamiento, el afecto y la conducta, con una desestructuración de la conciencia en el sentido de Ey. Los síntomas aparecen de forma muy aguda, aunque puede haber ciertos pródromos tales como ligeros cambios de humor, inquietud y logorrea, junto a discretas alteraciones del sueño o incluso algunas vivencias autorreferenciales, siendo frecuente la presencia de insomnio total en los días previos a la eclosión del cuadro. Estas psicosis tienen una tendencia a la alternancia, es decir, una estructura polar muy característica, existiendo fluctuaciones entre cuadros de excitación con otros de retardo, con una remisión completa del episodio en un tiempo variable, que en general no excede de tres semanas, siendo frecuente que el paciente no guarde un recuerdo total de los síntomas, refiriendo que lo que sí recuerda lo vivencia de un modo análogo a los sueños. Es clara también la tendencia a la repetición de los episodios con buen pronóstico a largo plazo en el sentido de que no aparece deterioro, manteniéndose una buena adaptación familiar, social y laboral.

Deteniéndonos en los subtipos descritos por Leonhard, la psicosis de angustia-felicidad tiene como rasgos característicos la ansiedad intensa y fluctuante, 
como angustia desconfiada, oscilando con sentimientos de felicidad, éxtasis y placidez, delirios místico-religiosos, preocupación por la muerte y alucinaciones auditivas y visuales complejas. Frente a la experiencia del maniaco con franca intranquilidad motora, la experiencia de felicidad en las psicosis cicloides es estática, calmada y placentera. La psicosis confusional (incoherente-estuporosa) tiene como síntoma esencial un trastorno del pensamiento, que puede consistir en aceleración y ruptura de la línea directriz con verborrea que en ocasiones llega a la incoherencia, o bien puede estar muy lentificado. Aparecen también falsos reconocimientos, confusión mental y perplejidad o estupor. Por último, la psicosis de la motilidad (acinética-hipercinética) alterna fases de hipercinesia y de acinesia, siendo los síntomas más característicos las hipercinesias expresivas y pseudoexpresivas, los movimientos pseudoespontáneos y en cortocircuito, las paracinesias y la inmovilidad de tipo cataléptico con rigidez mímica y negativismo.

Perris (26) considera que en la clínica prácticamente no es posible distinguir estos tres tipos aisladamente, por lo que sería preferible hablar de las psicosis cicloides como un solo grupo. Barcia insiste en el hecho de la gran labilidad o variabilidad sintomatológica y la bipolaridad excitación-retardo presentes en estas psicosis, en cada episodio y entre diferentes episodios, considerándolo un aspecto esencial y el carácter diferenciador más importante de las psicosis cicloides respecto a otras formas de psicosis endógenas y reactivas. También considera, siguiendo a Leonhard, que un cuadro relativamente estable es incompatible con el diagnóstico de psicosis cicloide.

Existen unos criterios diagnósticos para este trastorno desarrollados por Perris y Brockington (27):

a) Condición psicótica aguda entre los 15 y los 50 años.

b) Comienzo repentino en unas cuantas horas o, como mucho, unos cuantos días.

c) Para el diagnóstico definitivo se requiere la concurrencia de, al menos, cuatro de los siguientes síntomas:

1. Confusión en algún grado, frecuentemente expresada como perplejidad.

2. Delirios con humor incongruente, de cualquier tipo, la mayoría de las veces de contenido persecutorio.

3. Experiencias alucinatorias, de cualquier tipo, a menudo relativas al temor a la muerte.

4. Una arrolladora, espantosa y persuasiva experiencia de ansiedad, no relacionada con una particular situación o circunstancia.

5. Profundos sentimientos de felicidad o éxtasis, la mayoría de las veces de tonalidad religiosa. 
6. Alteraciones de la motilidad de tipo acinético o hipercinético.

7. Una particular preocupación por la muerte.

8. Cambios de humor en origen, no lo bastante pronunciados como para justificar el diagnóstico de trastorno afectivo.

d) No hay una constelación sintomatológica fija, por el contrario, los síntomas pueden cambiar frecuentemente en el curso del mismo episodio, desde luego mostrando características bipolares.

En cuanto a la cuestión del tratamiento, el hecho de que este diagnóstico sea poco aceptado provoca que haya escasos estudios, pero los datos disponibles indican que las psicosis cicloides responden favorablemente al TEC y al tratamiento neuroléptico en la fase aguda. Leonhard se oponía al tratamiento crónico de mantenimiento para estos cuadros, aunque otros autores como Perris han sugerido la posibilidad de emplear litio solo o más raramente junto a un neuroléptico para disminuir la frecuencia de aparición de los episodios a largo plazo, lo que no se consigue si se emplean sólo neurolépticos. Barcia, entre otros, también señala que un tratamiento eficaz para la rápida remisión de los episodios agudos y para la prevención de recidivas es la combinación de butirofenonas con valproato o carbamazepina.

Para completar esta revisión, queremos referirnos a otra entidad clínica con importantes semejanzas con las psicosis cicloides: las psicosis atípicas. En la actualidad, la psiquiatría japonesa acepta este diagnóstico como un trastorno diferenciado. Según Mitsuda, el cuadro clínico se caracterizaría por manifestaciones caleidoscópicas y en rápida fluctuación. Inicialmente son frecuentes las alteraciones emocionales, luego estados oniroides y confusionales, con delirios y alucinaciones. La mayoría de las veces el curso es episódico o periódico y el pronóstico favorable. Sin embargo, a diferencia de las psicosis cicloides de Kleist y Leonhard, no se produce una remisión completa en todos los casos y sí puede haber una evolución hacia un síndrome defectual. Esto hace especialmente complicado el diagnóstico diferencial con la esquizofrenia en algunos casos. Mitsuda planteó una posible relación entre las psicosis atípicas y la epilepsia por semejanzas en el cuadro clínico, el curso, la elevada incidencia de alteraciones electroencefalográficas y una alta tasa de incidencia de epilepsia en familiares de pacientes.

\section{Conclusiones}

Después del trabajo de revisión bibliográfica que hemos llevado a cabo en relación con el posible diagnóstico del paciente descrito, hay varios puntos que han llamado nuestra atención y sobre los que querríamos hacer algún comentario 
adicional ya desde el punto de vista de nuestra opinión personal, así como plantear posibles controversias objeto tal vez de futuros debates.

En primer lugar, hemos comprobado cómo distintos autores, al hablar de las psicosis agudas, señalan que la mayoría de los casos llegan a una remisión plena. Esto nos hace preguntarnos qué ocurre con ese pequeño porcentaje de casos que no terminan en remisión. Nos parece que, en esos casos, posiblemente sería necesario recurrir a otros diagnósticos, probablemente esquizofrenia, para describir el cuadro. En nuestra opinión, e intentando acotar algo un terreno nosológico difícil, creemos que sería conveniente reservar el diagnóstico de psicosis aguda para cuadros con plena remisión y, en caso de que ésta no se produzca, buscar el diagnóstico de psicosis crónica que más se aproxime a los hechos clínicos.

También nos gustaría llamar la atención sobre un hecho mencionado ya por distintos autores clásicos, como es la gran dificultad que reviste el diagnóstico diferencial entre una psicosis aguda y un episodio maniaco. Dificultad que debemos intentar salvar con una exploración psicopatológica lo más minuciosa posible, que permita intentar discriminar si lo que predomina es la alteración global de la vida psíquica y el trastorno del pensamiento o bien el trastorno del afecto. Creemos que es posible que, en el momento actual, se esté produciendo un cierto sobrediagnóstico de manía, tal vez equivocando como tal cuadros que encajarían bien en el perfil de las llamadas psicosis agudas.

Otro punto en el que queremos detenernos, todavía en relación con el grupo de las psicosis agudas, es el tema siempre controvertido de las psicosis histéricas, al que ya hicimos referencia en un trabajo anterior (29). Tras la revisión realizada, opinamos que en base a las características clínicas que describen estos cuadros, no parece fácil diferenciar las psicosis histéricas como una entidad individualizada del conjunto de las psicosis agudas, siendo además en nuestro entorno un diagnóstico con una importante carga estigmatizante y peyorativa, incluso entre los propios profesionales. Creemos que, ante síntomas pseudopsicóticos de tipo delirante o alucinatorio, que no impresionen de carácter psicótico genuino y que aparezcan sin gran relevancia en un contexto clínico neurótico, se puede recurrir al socorrido diagnóstico de trastorno disociativo. Pero, en casos de sintomatología psicótica florida, aunque ciertos rasgos histéricos coloreen el cuadro, creemos más conveniente para el abordaje adecuado del paciente proceder a un diagnóstico de trastorno psicótico. No se nos escapa que es éste un tema complejo y que creemos que adolece de falta de estudios y reflexiones sobre el mismo que esperemos puedan ir produciéndose en el futuro.

Otra cuestión sujeta a cierta polémica es la relacionada con el diagnóstico y la ubicación nosológica de los llamados trastornos esquizoafectivos. Las clasificaciones actuales, como ya vimos, hablan de cuadros sin deterioro con mezcla de 
síntomas esquizofrénicos y afectivos, más hacia el primer grupo en el caso del DSM-IV-TR y más hacia el segundo en caso de la CIE-10. Uno de los problemas que apreciamos en esta categoría es su posible mal uso en la práctica clínica habitual, empleándose algunas veces no para diagnosticar pacientes que cumplen criterios de esquizofrenia y de trastorno afectivo, sino para pacientes que no llegan a cumplir claramente ni unos ni otros. Se emplea a veces para crear una especie de continuo entre las dos grandes psicosis endógenas, como una especie de «esquizofrenia leve» o de «trastorno bipolar grave», lejos creemos de la intención de los autores de las citadas clasificaciones. Pero, estando en nuestro derecho de no comulgar con ellas, sí creemos necesario que cuando usemos su terminología, lo hagamos con propiedad.

En cuanto al tema de las psicosis cicloides, en nuestra revisión hemos observado que se insiste especialmente en la necesidad de ciertas características del estado del paciente, de cierto cortejo sintomatológico en el momento transversal que incluye la gran variabilidad de dichos síntomas, la perplejidad o confusión, etc., descuidando tal vez el aspecto del curso, de la evolución longitudinal del trastorno. En nuestra opinión, creemos que es importante reivindicar el diagnóstico de psicosis cicloides, pero sobre todo por las características que presenta la evolución de estos cuadros: episodios psicóticos agudos, con o sin desencadenante aparente, que ceden de forma rápida y completa, pero que suelen recidivar aunque sin que llegue a aparecer un deterioro. Opinamos que el curso del trastorno del paciente comentado encaja perfectamente en esta descripción. No creemos que sea un trastorno especialmente escaso sino que, posiblemente, muchos pacientes que podrían encajar en él se hallan tal vez diagnosticados de trastorno esquizoafectivo, trastorno bipolar o incluso de trastorno disociativo, entre otros. La vieja controversia entre curso y estado, que ha acompañado toda la historia del concepto «esquizofrenia» se nos plantea también aquí, en cierto modo. En opinión de nuestro grupo, un diagnóstico basado principalmente, aunque no de forma exclusiva, en el curso puede ser el más útil a la hora de diferenciar a pacientes que muchas veces son similares en sus manifestaciones en un momento puntual de su evolución. Reservar el término de esquizofrenia para cuadros psicóticos crónicos con deterioro, o bien paranoia si, siendo también crónicos, no existe tal deterioro. Y diferenciar entre trastornos bipolares o psicosis cicloides si, ante la presencia de fases con recuperación plena y sin deterioro, predomina el aspecto afectivo o bien el trastorno delirante-alucinatorio con cierta afectación global de la vida psíquica.

Consideramos que poder emplear este diagnóstico de psicosis cicloides tiene importancia no sólo a nivel teórico o académico, sino práctico en el abordaje diario de nuestros pacientes. La investigación psiquiátrica actual está muy lejos todavía de poder responder las cuestiones acerca de las causas, desarrollo, pronóstico o trata- 
miento de las diferentes enfermedades mentales, y consideramos que una parte de la dificultad en encontrar estas respuestas estriba en que posiblemente se mezclan en nuestras categorías diagnósticas pacientes con trastornos muy diferentes. Creemos que los pacientes diagnosticables de psicosis cicloides son muy distintos a los que encajan en el concepto de esquizofrenia o trastorno bipolar, pero si no los separamos a la hora de llevar a cabo cualquier investigación, estaremos lejos de encontrar datos útiles para unos o para otros. Al no ser un diagnóstico reconocido oficialmente, tenemos muy pocos estudios sobre su epidemiología, características o tratamiento, lo que sin duda puede repercutir en que no se esté abordando a estos pacientes de la mejor manera posible, al no conocer con certeza cuestiones clave como, por ejemplo, si existe necesidad de terapéutica de mantenimiento o no, tema que enfrenta a distintos autores y que probablemente no podrá resolverse mientras no haya estudios amplios sobre pacientes con este trastorno. Estudios que necesitarán como condición previa que se llegue a un cierto reconocimiento del trastorno como tal.

Nuestra conclusión es que el diagnóstico de psicosis cicloide puede ser útil a la vez que necesario para describir a determinados pacientes con características similares y diferentes a otros grupos, lo que podrá permitir una mejor comprensión y terapéutica de unos y otros.

\section{BIBLIOGRAFÍA}

(1) OMS, Décima revisión de la Clasificación Internacional de las Enfermedades (CIE-10), Madrid, Meditor, 1992.

(2) Asociación Americana de Psiquiatría, Manual diagnóstico y estadístico de los trastornos mentales (DSM-IV-TR), Barcelona, Masson, 2002.

(3) Luque Luque, R., Psicosis agudas: análisis histórico, conceptual y clínico, en DíEZ Patricio, A.; Luque Luque, R. (eds.), Psicopatología de los síntomas psicóticos, Madrid, AEN/Estudios, 2006.

(4) Magnan, V., Leçons cliniques sur les maladies mentales, París, Bataille, 1893.

(5) Kraepelin, E., Tratado de Psiquiatría, octava edición (Edimburgo, Robertson, 1921).

(6) Bleuler, E., Demencia precoz. El grupo de las esquizofrenias, Buenos Aires, Hormé, 1969.

(7) Ey, H.; Bernard, P.; Brisset, Ch., Tratado de Psiquiatría, Barcelona, Masson, 1998.

(8) Ey, H., Estudios sobre los delirios, Madrid, Paz Montalvo, 1950.

(9) INSERM, «Classification française des troubles mentaux», Bulletin de l'Institut National de la Santé et de la Recherche Médicale, 1969, 24, pp. 1-29.

(10) Sommer, R., Diagnostik der Geisteskrankheiten, Viena y Leipzig, Urban und Schwarzenberg, 1894.

(11) Wimmer, A., Psykogene Sindssygdomsformer, St. Hans Hospital 1816-1916, Copenhague, Jubilee Publication, Gad, 1916, pp. 85-216.

(12) Strömgren, E., «The Development of the Concept of Reactive Psychoses», British Journal of Psychiatry, 1989, 154, pp. 47-50. 
(13) Strömgren, E., «Psychogenic Psychoses», en Hirsch, S. R.; ShePherd, M. (eds.), Themes and Variations in European Psychiatry, Charlottesville, University Press of Virginia, 1974.

(14) Faergeman, P., Psychogenic Psychoses. A Description and Follow-up of Psychoses Following Psychological Stress, Londres, Butterworths, 1963.

(15) Hollender, M. H.; Hirsch, S. J., «Hysterical Psychosis», American Journal of Psychiatry, 1964, 120, pp. 1.066-1.074.

(16) Modestin, J.; Sonderegger, P.; ERni, T., «Follow-up Study of Hysterical Psychosis, Reactive/Psychogenic Psychosis, and Schizophrenia», Comprehensive Psychiatry, 2001, 42, pp. 51-56.

(17) Langfeldt, G., The Schizophreniform States, Copenhague, Munksgaard, 1939.

(18) KASAnIN, J., «Acute Schizoaffective Psychosis», American Journal of Psychiatry, 1933, 13, pp. 97-126.

(19) Castilla del Pino, C., Introducción a la psiquiatría. 2. Psiquiatría general. Psiquiatría clínica, Madrid, Alianza, 1980.

(20) SCHNeIDER, K., Patopsicología clínica, Madrid, Paz Montalvo, 1970.

(21) Barcia, D., Psicosis cicloides, Madrid, Triacastela, 1998.

(22) Barcia, D., Tratado de Psiquiatría, Madrid, Arán, 2000.

(23) KleIst, K., «Über Cycloide, Paranoide und Epileptoide Psychosen und über die Frage der Degenerationpsychosen», Schweizer Archiv für Neurologie und Psychiatrie, 1928, 23, pp. 1-17.

(24) LeOnHARD, K., «Cycloid Psychoses-Endogenous Psychoses Which Are Neither Schizophrenic nor Manic Depressive», Journal of Mental Science, 1961, 107, pp. 632-648.

(25) Leonhard, K., Classification of Endogenous Psychoses and Their Differential Etiology, Viena y Nueva York, Beckman, 1999.

(26) Perris, C., «Leonhard and the Cycloid Psychoses», en Berrios, G. E.; Porter, R. (eds.), A History of Clinical Psychiatry. The Origin and History of Psychiatric Disorders, Londres, Athlone, 1995.

(27) Perris, C.; Brockington, I. F., «Cycloid Psychoses and their Relation to the Major Psychoses», en Perris, C.; Struwe, G.; Jansson, B. (eds.), Biological Psychiatry, Amsterdam, Elsevier, 1981.

(28) Mitsuda, H., «The Concept of 'Atypical Psychosis' from the Aspect of Clinical genetics», Acta Psychiatrica Scandinavica, 1965, 41, pp. 372-377.

(29) García-VAldecasas CAMPElo, J., y otros, «Encuadre nosológico de la "psicosis histérica" versus psicosis agudas: Revisión a propósito de un caso», Revista de la AEN, 2005, XXV, 95, pp. 9-23.

* José García-Valdecasas Campelo, psiquiatra; Amaia Vispe Astola, enfermera especialista en Salud Mental; Desirée Díaz Melián, M.I.R., psiquiatría; Marta Martín Olmos, MIR Servicio de Psiquiatría. Hospital Universitario de Canarias. Consorcio Sanitario de Tenerife.

Correspondencia: jose_valdecasas@hotmail.com

** Fecha de recepción: 18-X-06. 